\title{
Characteristic equations with solutions that contain nature's golden ratio
}

\author{
B. T. Rosson \\ Department of Civil Engineering, Florida Atlantic University, USA
}

\begin{abstract}
It has been shown that certain characteristic equations of eigenproblems produce results in which the golden ratio is revealed. The characteristic equations that produce these results are developed for application to a broad range of physical systems. The resulting equations provide the background to understand why these results are obtained and the physical system requirements needed to produce them. Two physical systems that typically involve eigenproblems in structural mechanics are used to illustrate the use of the equations. The analysis of natural frequencies and principal stresses involve characteristic equations of the general form presented in the paper. Examples are presented that demonstrate how the equations can be used to determine the physical conditions that are necessary to produce golden ratio solutions, and also how they may be utilized to study the dimensions of the natural world.
\end{abstract}

Keywords: golden ratio, characteristic equation, eigenproblem, Fibonacci series, frequency, principal stress, dimensions.

\section{Introduction}

A few physical systems have been discovered that reveal the golden ratio in the solution of the equations that predict their natural behavior or response to external action. Since the vast majority of physical systems do not reveal such results, one seems left to discover these unique systems somewhat serendipitously. It is to be expected that systems with physical properties of golden proportion will reveal these results, but for physical systems that do not, finding the conditions to produce them are not so obvious. For instance, the structural system in Fig. 1 has no physical properties that contain the golden proportion, but Moorman and Goff [1] have shown that the system's natural 


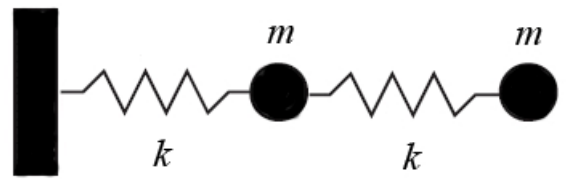

Figure 1: Undamped two-degree-of-freedom system with two identical masses and springs.

frequencies are $\phi \omega_{o}$ and $\Phi \omega_{o}$, in which $\phi=(\sqrt{5}-1) / 2, \Phi=(\sqrt{5}+1) / 2$ and $\omega_{o}=\sqrt{k / m}$.

Borges [2] has shown that the plane stress condition of $\sigma_{x}=\tau_{x y}$ and $\sigma_{y}=0$ produces principal stresses $\sigma_{1}=\Phi \sigma_{x}$ and $\sigma_{2}=-\phi \sigma_{x}$. From a study of the resonance in wind tunnels with ventilated walls, Mokry [3] has shown by use of acoustic wave theory that $\phi$ determines the critical Mach number below which refraction is possible and above which total reflection takes place. By knowing $a$ priori a unique set of polynomials that have roots containing golden ratios, one can begin to explain how these results were obtained.

\section{Polynomials with golden ratio roots}

It will be shown that the following general form equation

$$
\mathrm{z}^{r}-C_{\mathrm{a}} \mathrm{z}^{s}+C_{\mathrm{b}}=0 \text { for } r>s
$$

will produce golden ratio roots for coefficients $C_{\mathrm{a}}$ and $C_{\mathrm{b}}$ corresponding to given $r$ and $s$ conditions. (For ease of presentation, only the equations with integer coefficients will be presented in this paper.)

Two adjacent numbers in the Fibonacci series can be used to express the value of $\Phi$ and $\phi$ raised to the power of $n$, for $n=0,1,2,3,4, \ldots$ etc.

$$
\begin{aligned}
& \Phi^{n}=\mathrm{F}_{n-1}+\mathrm{F}_{n} \Phi \\
& \phi^{n}=(-1)^{n}\left(\mathrm{~F}_{n-1}-\mathrm{F}_{n} \phi\right)
\end{aligned}
$$

Using eqns (1) and (2), $\Phi$ is revealed as a solution in the following linear equation

$$
\left(\mathrm{F}_{r-1}+\mathrm{F}_{r} \Phi\right)-C_{1}\left(\mathrm{~F}_{s-1}+\mathrm{F}_{s} \Phi\right)+C_{2}=0
$$

The form of this equation allows for a direct determination of the coefficients that satisfy eqn (1) for specified $r$ and $s$ conditions. In order for the left side of eqn (4) to equal zero, $\mathrm{F}_{r}=C_{1} \mathrm{~F}_{s}$ and $C_{1} \mathrm{~F}_{s-1}-\mathrm{F}_{r-1}=C_{2}$.

The $r$ and $s$ conditions that provide for integer coefficients $C_{1}$ and $C_{2}$ in eqn (4) are given in Table 1. (Note that the results are only presented for $2 \leq r \leq 8$.) 
The same equations that reveal $\Phi$ as a root also produce $-\phi$ as a root. This is because

$$
(-\phi)^{n}=\mathrm{F}_{n-1}+\mathrm{F}_{n}(-\phi)
$$

is of the same form as eqn (2) and when used with eqn (1), it produces the same $C_{1}$ and $C_{2}$ coefficients for the given $r$ and $s$ conditions in Table 1. Refer to Fig. 2 for an illustration of several polynomials with coefficients $C_{1}$ and $C_{2}$ that have golden ratio roots at $\Phi$ and $-\phi$.

Table 1: Exponents and integer coefficients of eqn (1) with golden ratio roots.

\begin{tabular}{|cc|cc|cc|}
\hline $\boldsymbol{r}$ & $\boldsymbol{S}$ & $\boldsymbol{C}_{\mathbf{1}}$ & $\boldsymbol{C}_{\mathbf{2}}$ & $\boldsymbol{C}_{\mathbf{3}}$ & $\boldsymbol{C}_{\mathbf{4}}$ \\
\hline 2 & 1 & 1 & -1 & -1 & -1 \\
3 & 2 & 2 & 1 & -2 & -1 \\
3 & 1 & 2 & -1 & 2 & 1 \\
4 & 2 & 3 & 1 & 3 & 1 \\
4 & 1 & 3 & -2 & -3 & -2 \\
5 & 2 & 5 & 2 & -5 & -2 \\
5 & 1 & 5 & -3 & 5 & 3 \\
6 & 3 & 4 & -1 & -4 & -1 \\
6 & 2 & 8 & 3 & 8 & 3 \\
6 & 1 & 8 & -5 & -8 & -5 \\
7 & 2 & 13 & 5 & -13 & -5 \\
7 & 1 & 13 & -8 & 13 & 8 \\
8 & 4 & 7 & 1 & 7 & 1 \\
8 & 2 & 21 & 8 & 21 & 8 \\
8 & 1 & 21 & -13 & -21 & -13 \\
\hline
\end{tabular}

Using eqns (1) and (3), $\phi$ is revealed as a solution in the following linear equation

$$
(-1)^{r}\left(\mathrm{~F}_{r-1}-\mathrm{F}_{r} \phi\right)-C_{3}(-1)^{s}\left(\mathrm{~F}_{s-1}-\mathrm{F}_{s} \phi\right)+C_{4}=0
$$

The coefficients can be obtained directly from $(-1)^{r} \mathrm{~F}_{r}=C_{3}(-1)^{s} \mathrm{~F}_{s}$ and $C_{3}(-1)^{r} \mathrm{~F}_{r-1}-(-1)^{s} \mathrm{~F}_{s-1}=C_{4}$. The $r$ and $s$ conditions that provide for integer coefficients $C_{3}$ and $C_{4}$ in eqn (6) are presented in Table 1. 


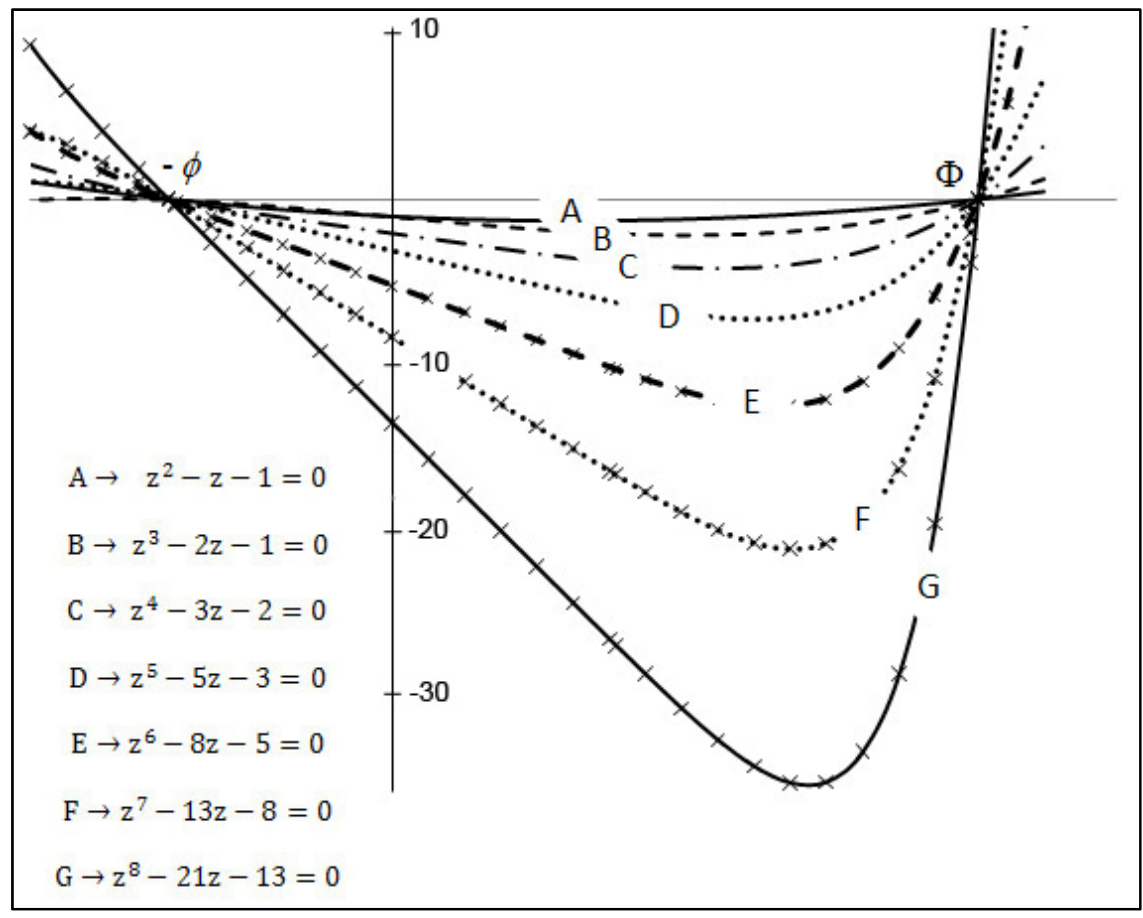

Figure 2: $\quad$ Examples of polynomials with coefficients $C_{1}$ and $C_{2}$ producing roots at $\Phi$ and - $\phi$.

Similarly as before, the same equations that reveal $\phi$ as a root also produce $-\Phi$ as a root. This is because

$$
(-\Phi)^{n}=(-1)^{n}\left(\mathrm{~F}_{n-1}-\mathrm{F}_{n}(-\Phi)\right)
$$

is of the same form as eqn (3) and when used with eqn (1), it produces the same $C_{3}$ and $C_{4}$ coefficients for the given $r$ and $s$ conditions in Table 1.

Notice in Table 1 that $\Phi$ and $\phi$ are solutions of the same polynomials when both $r$ and $s$ are even numbers. As illustrated in Fig. 3, these equations also produce roots at $-\Phi$ and $-\phi$.

\section{Natural frequencies}

Moorman and Goff [1] have shown that the natural frequencies of the physical system in Fig. 1 are $\phi \omega_{o}$ and $\Phi \omega_{o}$. The characteristic equation used to obtain these results is

$$
\omega^{4}-3 \omega_{o}^{2} \omega^{2}+\omega_{o}^{4}=0
$$




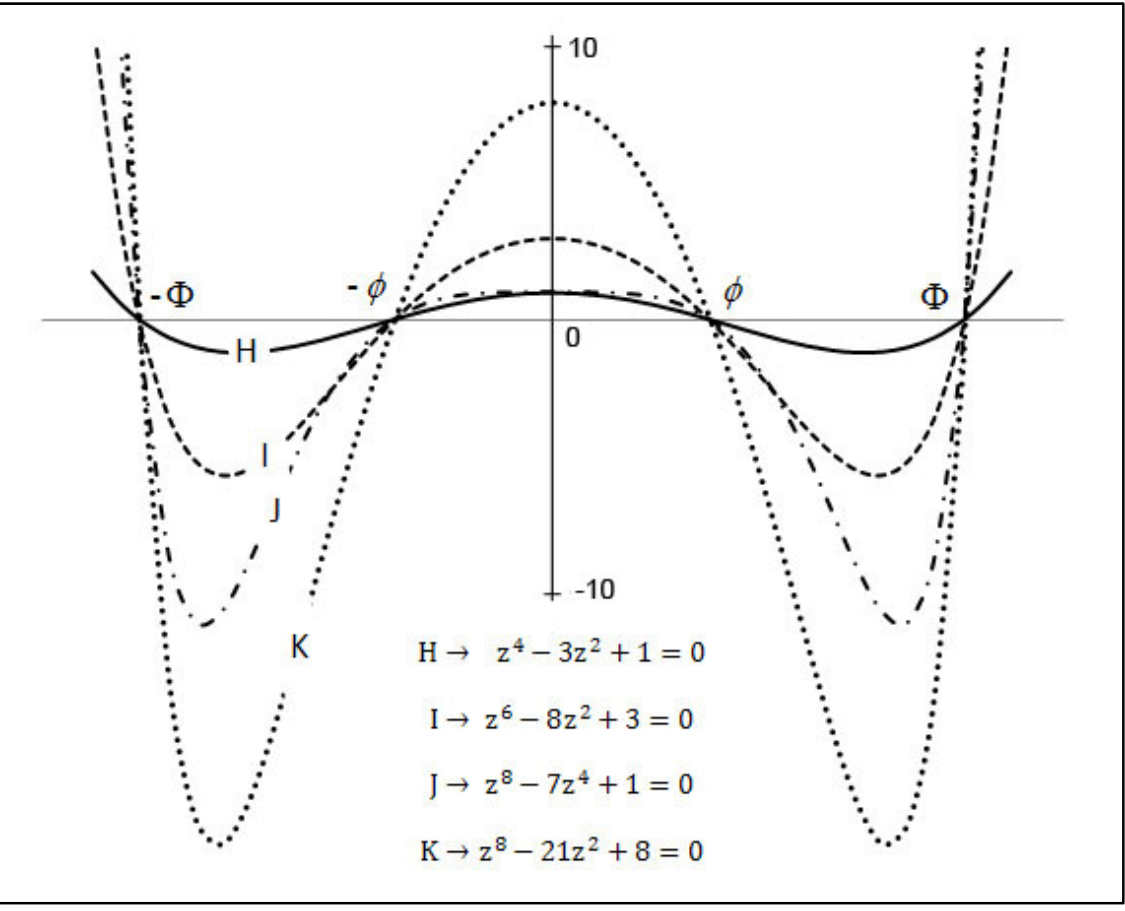

Figure 3: $\quad$ Examples of polynomials with even number exponents producing roots at $\Phi, \phi,-\phi$ and $-\Phi$.

Considering that $\omega_{o}=1$ is a possible condition of $k$ and $m$, then it is easily recognizable from polynomial $\mathrm{H}$ in Fig. 3 that the golden ratios would be revealed in their solution of eqn (8).

This idea can be expanded by considering the physical system in Fig. 4. This is a similar two-degree-of-freedom system, but the masses $\left(m_{1}\right.$ and $\left.m_{2}\right)$ and the stiffnesses $\left(k_{1}, k_{2}\right.$ and $\left.k_{3}\right)$ are allowed to vary. The system's characteristic equation was found by Hartog [4] to be

$$
\omega^{4}-\left(\frac{k_{1}+k_{3}}{m_{1}}+\frac{k_{2}+k_{3}}{m_{2}}\right) \omega^{2}+\left(\frac{k_{1} k_{2}+k_{2} k_{3}+k_{1} k_{3}}{m_{1} m_{2}}\right)=0
$$

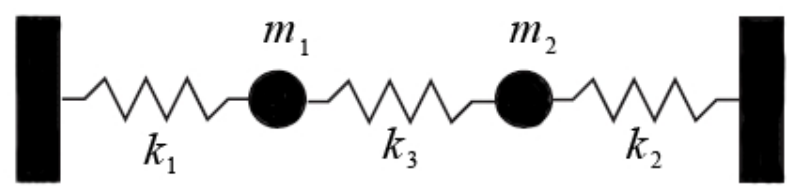

Figure 4: Undamped two-degree-of-freedom system with two unequal masses and three unequal springs. 
Comparing eqn (9) with eqn (8), it is evident that there are mass and stiffness conditions that yield the same natural frequencies $\phi \omega_{o}$ and $\Phi \omega_{o}$ when

$$
\left(\frac{k_{1}+k_{3}}{m_{1}}+\frac{k_{2}+k_{3}}{m_{2}}\right)=3 \omega_{o}^{2}
$$

and

$$
\left(\frac{k_{1} k_{2}+k_{2} k_{3}+k_{1} k_{3}}{m_{1} m_{2}}\right)=\omega_{o}^{4}
$$

There are many combinations of mass and stiffness that satisfy these equations, but for the condition when $k=k_{1}=k_{2}=k_{3} / \beta$ and $m=m_{1}=$ $m_{2} / \mu$ the characteristic equation becomes

$$
\omega^{4}-\frac{(1+\mu)(1+\beta)}{\mu} \omega_{o}^{2} \omega^{2}+\left(\frac{1+2 \beta}{\mu}\right) \omega_{o}^{4}=0
$$

The conditions of $\beta$ and $\mu$ that reduce eqn (12) to eqn (8), and thus produce natural frequencies $\phi \omega_{o}$ and $\Phi \omega_{o}$, are $\beta=(1+\sqrt{3}) / 2$ and $\mu=2+\sqrt{3}$.

Example 1: Let the physical system in Fig. 4 have the following properties:

$$
k_{1}=k_{2}=30 \mathrm{~N} / \mathrm{m} \quad k_{3}=41 \mathrm{~N} / \mathrm{m}_{1}=30 \mathrm{~kg} \quad m_{2}=112 \mathrm{~kg}
$$

Substituting these values into eqn. (9) yields the following

$$
\omega^{4}-\left(\frac{30+41}{30}+\frac{30+41}{112}\right) \omega^{2}+\left(\frac{30 \cdot 30+30 \cdot 41+30 \cdot 41}{30 \cdot 112}\right)=0
$$

Solving for the positive roots of this equation yields the following natural frequencies

$$
\omega_{1}=0.618 \mathrm{rad} / \mathrm{sec} \quad \omega_{2}=1.618 \mathrm{rad} / \mathrm{sec}
$$

These results were obtained because the stiffness and mass ratios were chosen to closely approximate the exact $\beta$ and $\mu$ values given above.

\section{Principal stresses}

Borges [2] provided an interesting example in which the two principal stresses $\sigma_{1}=\Phi \sigma_{x}$ and $\sigma_{2}=-\phi \sigma_{x}$ are revealed for a prescribed plane stress condition of $\sigma_{x}=\tau_{x y}$ and $\sigma_{y}=0$. In order to explore how golden ratios can be found for other stress conditions, it is necessary to begin with the Cauchy stress tensor

$$
\sigma_{i j}=\left[\begin{array}{ccc}
\sigma_{x} & \tau_{x y} & \tau_{x z} \\
\tau_{y x} & \sigma_{y} & \tau_{y z} \\
\tau_{z x} & \tau_{z y} & \sigma_{z}
\end{array}\right]
$$


The three normal stresses and six shear stresses are shown in Fig. 5. The characteristic equation that is commonly used to obtain the principal stresses is

$$
\sigma^{3}-I_{1} \sigma^{2}+I_{2} \sigma-I_{3}=0
$$

The stress invariants $I_{1}, I_{2}$ and $I_{3}[5]$ are written as a function of the stresses as

$$
\begin{gathered}
I_{1}=\sigma_{x}+\sigma_{y}+\sigma_{z} \\
I_{2}=\sigma_{x} \sigma_{y}+\sigma_{y} \sigma_{z}+\sigma_{x} \sigma_{z}-\tau_{x y}^{2}-\tau_{y z}^{2}-\tau_{x z}^{2} \\
I_{3}=\sigma_{x} \sigma_{y} \sigma_{z}+2 \tau_{x y} \tau_{y z} \tau_{x z}-\sigma_{x} \tau_{y z}^{2}-\sigma_{y} \tau_{x z}^{2}-\sigma_{z} \tau_{x y}^{2}
\end{gathered}
$$

The stress invariants can also be expressed as a function of the principal stresses as

$$
\begin{gathered}
I_{1}=\sigma_{1}+\sigma_{2}+\sigma_{3} \\
I_{2}=\sigma_{1} \sigma_{2}+\sigma_{2} \sigma_{3}+\sigma_{1} \sigma_{3} \\
I_{3}=\sigma_{1} \sigma_{2} \sigma_{3}
\end{gathered}
$$

For the plane stress condition $\sigma_{z}=\tau_{x z}=\tau_{y z}=0$, the characteristic equation becomes

$$
\sigma^{2}-I_{1} \sigma+I_{2}=0
$$

and the stress invariants become

$$
\begin{aligned}
& I_{1}=\sigma_{x}+\sigma_{y}=\sigma_{1}+\sigma_{2} \\
& I_{2}=\sigma_{x} \sigma_{y}-\tau_{x y}^{2}=\sigma_{1} \sigma_{2}
\end{aligned}
$$

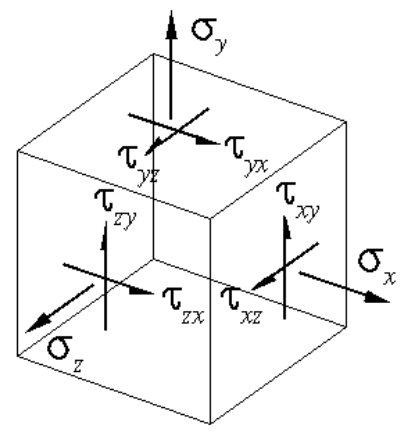

Figure 5: Cauchy stress components in three dimensions. 
Returning to the plane stress example given by Borges [2] in which $\sigma_{x}=\tau_{x y}$ and $\sigma_{y}=0$, the stress invariants $I_{1}=\sigma_{x}$ and $I_{2}=-\sigma_{x}^{2}$. Using eqn (21), the characteristic equation is

$$
\sigma^{2}-\sigma_{x} \sigma-\sigma_{x}^{2}=0
$$

As expected, solving for the roots of this quadratic equation, or using eqns (22) and (23), the principal stresses are found to be $\sigma_{1}=\Phi \sigma_{x}$ and $\sigma_{2}=-\phi \sigma_{x}$. With this background, one can now begin to investigate other more complex stress conditions that produce principal stresses in which the golden ratio is revealed.

Example 2: Find a Cauchy stress tensor with principal stresses of $\sigma_{1}=\Phi \mathrm{MPa}$, $\sigma_{2}=1 \mathrm{MPa}$, and $\sigma_{3}=-\phi \mathrm{MPa}$. Using eqns (15) through (20), the stress invariants are

$$
\begin{gathered}
I_{1}=\sigma_{x}+\sigma_{y}+\sigma_{z}=\Phi+1-\phi=2 \\
I_{2}=\sigma_{x} \sigma_{y}+\sigma_{y} \sigma_{z}+\sigma_{x} \sigma_{z}-\tau_{x y}^{2}-\tau_{y z}^{2}-\tau_{x z}^{2}=\Phi \cdot 1-1 \cdot \phi+\Phi \cdot \phi=0 \\
I_{3}=\sigma_{x} \sigma_{y} \sigma_{z}+2 \tau_{x y} \tau_{y z} \tau_{x z}-\sigma_{x} \tau_{y z}^{2}-\sigma_{y} \tau_{x z}^{2}-\sigma_{z} \tau_{x y}^{2}=-\phi \cdot \Phi \cdot 1=-1
\end{gathered}
$$

There are many normal and shear stress conditions that satisfy the three stress invariant conditions of $I_{1}=2, I_{2}=0$ and $I_{3}=-1$. To satisfy the first condition of $I_{1}=2$, one solution is found when $\sigma_{x}=\sigma_{y}=1 \mathrm{MPa}$ and $\sigma_{z}=0$. Using these normal stresses, the shear stresses that satisfy the $I_{2}$ and $I_{3}$ conditions are found using

$$
\begin{gathered}
I_{2}=1-\tau_{x y}^{2}-\tau_{y z}^{2}-\tau_{x z}^{2}=0 \\
I_{3}=2 \tau_{x y} \tau_{y z} \tau_{x z}-\tau_{y z}^{2}-\tau_{x z}^{2}=-1
\end{gathered}
$$

When $\tau_{x y}=0$, both equations become identical, and the following relationship is found for the two remaining shear stresses.

$$
\tau_{y z}^{2}+\tau_{x z}^{2}=1
$$

One solution to this equation is found when $\tau_{y z}=\tau_{x z}=\sqrt{1 / 2} \mathrm{MPa}$. Thus a Cauchy stress tensor with principal stresses $\sigma_{1}=\Phi \mathrm{MPa}, \sigma_{2}=1 \mathrm{MPa}$, and $\sigma_{3}=-\phi \mathrm{MPa}$ is given as

$$
\sigma_{i j}=\left[\begin{array}{ccc}
1 & 0 & \sqrt{1 / 2} \\
0 & 1 & \sqrt{1 / 2} \\
\sqrt{1 / 2} & \sqrt{1 / 2} & 0
\end{array}\right] \mathrm{MPa}
$$

As when considering any three dimensional stress state, other Cauchy stress tensors produce the same principal stresses. This example illustrates the steps that can be followed to obtain one such stress state in which the principal stresses have golden ratio values. 
The characteristic equation is unique to this problem and is obtained using the three stress invariants and eqn (14).

$$
\sigma^{3}-2 \sigma^{2}+1=0
$$

The polynomial in Table 1 of $r=3$ and $s=2$ is evident with this characteristic equation.

\section{Use of the polynomials to study dimensions in nature}

Although the primary purpose of this paper is to bring about a broader understanding of how golden ratios are revealed in the solution of characteristic equations, the polynomials can also be used for other purposes. Since the equations inherently have as their roots dimensions that are found in the natural world, it is perhaps not surprising that the equations, for instance, can be employed to investigate the dimensions of flora and fauna. By way of two examples, the polynomials $\mathrm{H}$ and $\mathrm{I}\left(z^{4}-3 z^{2}+1=0\right.$ and $z^{6}-8 z^{2}+3=0$, respectively) in Fig. 2 will be used to illustrate how they may be used in this manner.

Both equations are even functions, thus the first example will involve physical characteristics in which there is at least one axis of symmetry. The face of an owl has a striking symmetry, so it was chosen for further study. As illustrated in Fig. 6, the curves seem to intersect at key features of its anatomy.

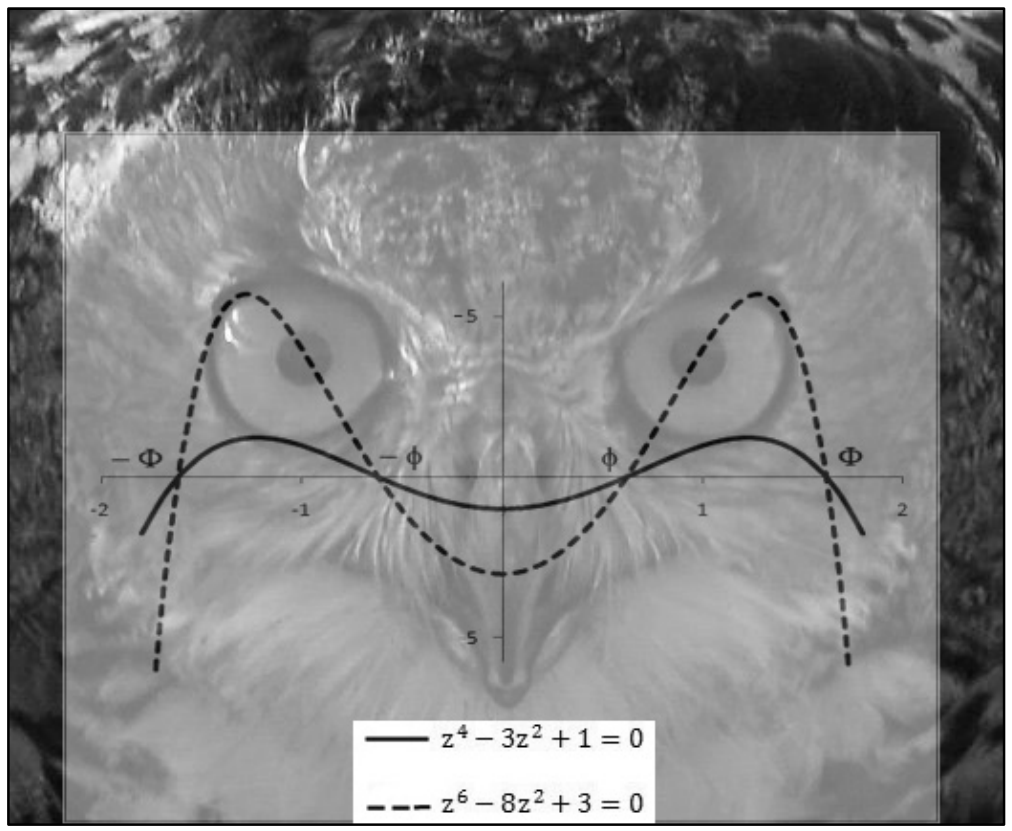

Figure 6: Owl face with superimposed graph of two polynomials. 
The solid line $(\mathrm{H})$ passes just below each eye and nostril, while at the same horizontal and vertical scale, the dashed line (I) intersects each pupil of the eye. Also at this same scale, the inside of each eye is found to be at $\phi$ and $-\phi$.

The second example uses the same equations to investigate the dimensions of a leaf. As illustrated in Fig.7, when the graph is scaled such $\phi$ and $-\phi$ intersect at the juncture of the veins and midrib, the third intersection occurs at $-\Phi$.

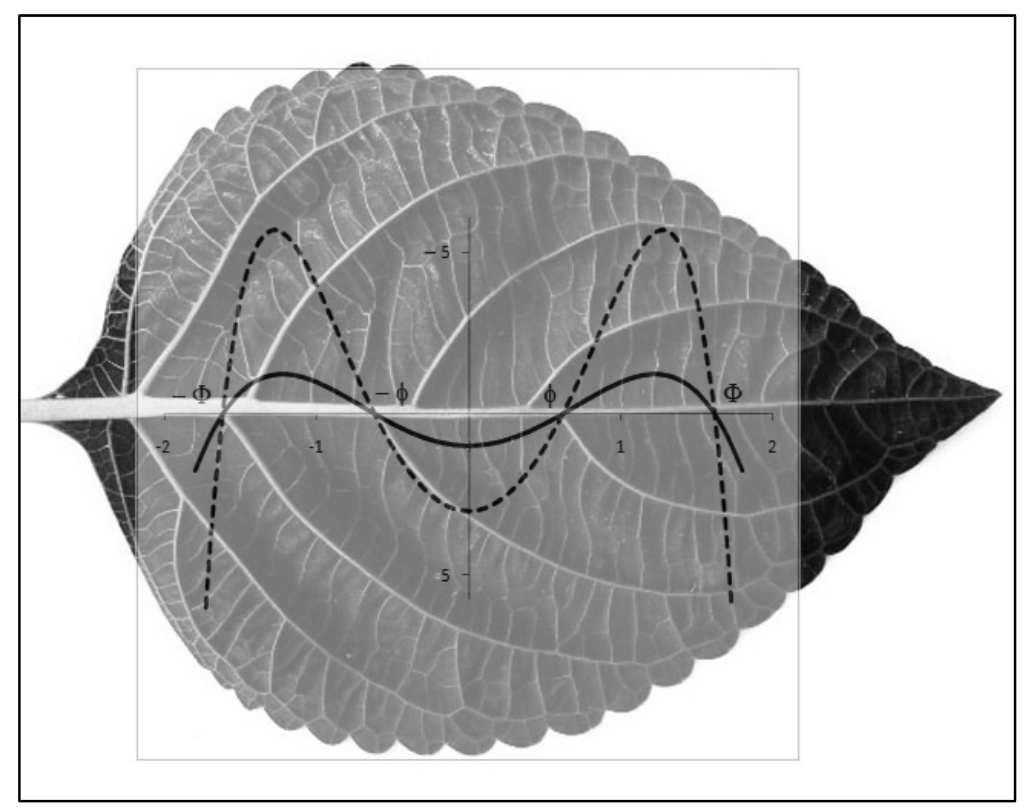

Figure 7: Leaf with superimposed graph of two polynomials.

\section{Concluding remarks}

It has been shown that characteristic equations of the general form in eqn (1) produce at least two golden ratio solutions. One is not left to find these solutions by chance, but now, with a broader understanding of the origin of the equations, golden ratio solutions will always be revealed when the polynomials in Table 1 are either intentionally utilized or found to exist as a result of scientific inquiry. When the polynomials are intentionally used, there is no special physical meaning inherent in the solution of the characteristic equations. Indeed the two examples in the paper illustrate how their intentional use will yield golden ratio solutions, but that no apparent significance can be attributed to either physical system. However, the golden ratio as revealed in the discovery by Mokry [3] concerning the critical Mach number and by Heyrovska [6] concerning inter- 
atomic distances, the polynomial of $r=2$ and $s=1$ is found to naturally arise from their research and thus contextually has greater meaning.

The use of the polynomials to study the dimensions of the natural world is preliminary. Perhaps the results are coincidental, but perhaps they are not, and like the logarithmic spiral, they reveal yet one more way to appreciate nature's wonder and beauty.

\section{References}

[1] Moorman, C. M., and Goff, J. E., Golden ratio in a coupled-oscillator problem. European Journal of Physics, 28, pp. 897-902, 2007.

[2] Borges, R. F., The phi code in nature, architecture and engineering. Design and Nature II, WIT Press: Southampton, pp.401-409, 2004.

[3] Mokry, M., Encounters with the golden ratio in fluid dynamics. Design and Nature IV, WIT Press: Southampton, 114, pp. 119-128, 2008.

[4] Hartog, D., Mechanical Vibrations, Dover Publications: Mineola, N.Y., pp. 79-81, 1985.

[5] Timoshenko, S.P., and Goodier, J.N., Theory of Elasticity, McGraw-Hill: New York, pp. 223-224, 1970.

[6] Heyrovska. R., Golden sections of inter-atomic distances as exact ionic radii of atoms. Nature Precedings, Macmillan Publishers Ltd., pp. 1-15, March 2009 . 